\title{
Composite Broadcasting and Ranging via a Satellite Dual-Frequency MPPSK System
}

\author{
Yu Yao, Lenan Wu, and Jiwu Wang \\ School of Information Science and Engineering, Southeast University, Nanjing 210096, China \\ Correspondence should be addressed to Yu Yao; 1057604987@qq.com
}

Received 18 May 2013; Revised 11 September 2013; Accepted 11 October 2013

Academic Editor: Saeed Balochian

Copyright (C) 2013 Yu Yao et al. This is an open access article distributed under the Creative Commons Attribution License, which permits unrestricted use, distribution, and reproduction in any medium, provided the original work is properly cited.

\begin{abstract}
Since digital video broadcasting via satellite (DVB-S) signals are "inefficient", regarding the amount of information they convey on the bandwidth they occupy, a joint broadcasting and ranging system would constitute a unique platform for future digital video broadcasting satellite services effecting the essential tasks of satellite navigation system and direct to home (DTH) services, in terms of both spectrum efficiency and cost effectiveness. In this paper, the design of dual frequency M-ary position phase shift keying (MPPSK) system which is suitable for, respectively, performing both data transmission and range measurement is proposed. The approach is based on MPPSK modulation waveforms utilized in digital video broadcasting. In particular, requirements that allow for employing such signals for range measurements with high accuracy and high range are investigated. Also, the relationship between the frequency difference of dual frequency MPPSK system and range accuracy is discussed. Moreover, the selection of MPPSK modulation parameter for data rate and ranging is considered. In addition to theoretical considerations, the paper presents system simulations and measurement results of new systems, demonstrating the high spectral utilization of integrated broadcasting and ranging applications.
\end{abstract}

\section{Introduction}

Direct to home (DTH) services via satellite are particularly affected by power limitation, which weakens its ability of antinoise and antiinterference. Traditionally, power limitation is the main design objective rather than spectrum efficiency, so DVB-S system uses QPSK modulation [1]. However, the spectrum resource in the air has become more occupied, with rapid development of digital video and audio broadcasting. To achieve a very high spectrum efficiency without excessively penalizing the power efficiency, the modulation technique named M-ary position phase shift keying (MPPSK) was proposed, which is a kind of transmission technique with efficient-bandwidth and high data rate $[2,3]$.

Compared to EBPSK [4], MPPSK utilizes M-ary information symbols to directly control the positions of phase transition of sinusoidal carrier in each symbol cycle. These techniques offer a number of advantages, such as ultranarrow bandwidth, very high transmission efficiency, and adjustable data rate. Different from QPSK, MPPSK preserves a strong carrier within its RF spectra, because only a small portion of sine carrier is changed. Meanwhile, the receiver can extract the tiny modulation information to achieve demodulation while applying an extremely narrow passband filter named digital impacting filter (DIF). References $[5,6]$ explained the special mechanism of DIF elaborately. Continuous wave $(\mathrm{CW})$ ranging has been demonstrated potential for providing precise detection results; however, they suffer from large range ambiguity [7]. Dual frequency CW ranging has a contradiction between the accuracy and range of measurement $[8,9]$.

In the paper, a dual frequency MPPSK system is creatively proposed to overcome the ranging ambiguity. At the same time, the system is used for the digital video broadcasting satellite services. Such a kind of platform would offer unique possibilities for novel system concepts and applications. Even more important, by using the dual frequency MPPSK waveform for both applications, the occupied spectrum would be used very efficiently and both applications could be operated, respectively, which would guarantee availability of both 


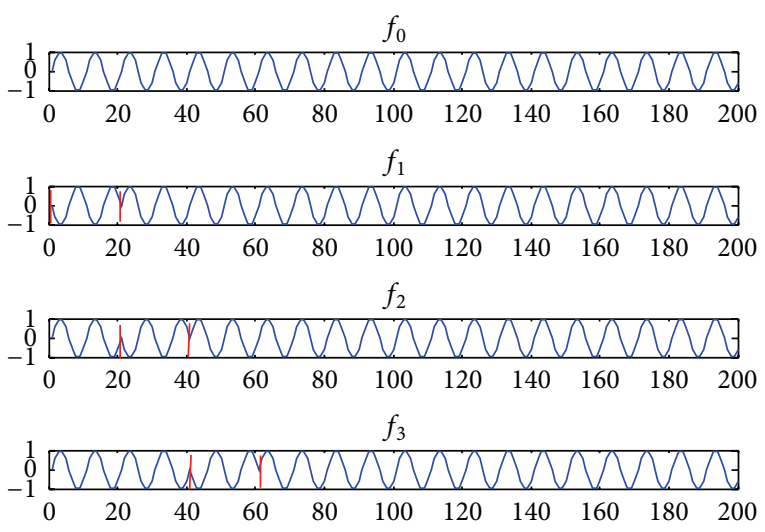

FIGURE 1: 4 PPSK modulated waveforms.

functions, and help to partially overcome the limitation of spectral resources. Such a system will provide two functions on a single hardware platform of MPPSK modems.

The rest of this paper is organized as follows: Section 2 introduces MPPSK modulation and demodulation, including the waveform character and the performance of impacting filters. Section 3 illustrates principle of dual frequency CW ranging. In Section 4, a block diagram of the dual frequency MPPSK broadcasting and receiving system is partly described, and also, two working modes are established. Some indicative simulation results and performance analysis are presented in Section 5. And finally, Section 6 gives the conclusion of the paper.

\section{MPPSK Modem}

2.1. MPPSK Modulation. The modulated MPPSK signals are defined by [2], and the simplified expression is presented in the paper as follows:

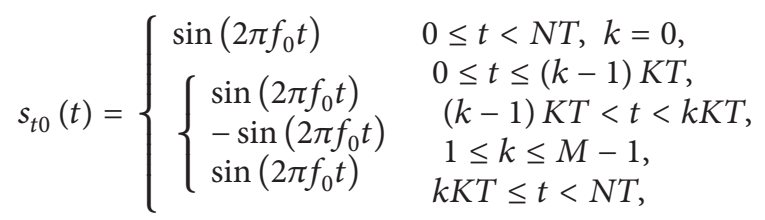

where $f_{0}$ and $T$ represent the carrier frequency and the carrier period, respectively. $K$ and $N$ stand for the number of the carrier period in each time slot and the number of the carrier period in each symbol, respectively. $N / K$ means the slot number in each symbol, and $m(m=0,1, \ldots, M-1)$ is M-ary $(M \geq 2)$ source symbol. The waveforms of 4PPSK modulation are illustrated as in Figure 1. The coefficient for the abscissa axis is the index of a certain sample point. Setting $K=2, N=20$.

The modulation waveform for symbol " 0 " is sinusoidal wave as shown in Figure 1. Figure 1 also illustrates the modulation waveform for symbol " 1 " with the phase hopping during the first two carrier period (from 0 to 20), the next (from 20 to 40 ) is for symbol " 2 ", and last (from 40 to 60 ) is 3 for symbol “ 3 ”.
MPPSK modulation generator is a new analog-digitalmixed type. Pulse train MPPSK modulator is illustrated by [3]. The modulating process of 4 PPSK signal in carrier frequency $f_{1}$ is shown in Figure 2 .

The next step is to explain the processes more clearly, and the output of a sinewave oscillator with frequency $f_{1}$ is divided into two branches: the upper is direct output with no phase shift, while the lower is phase reversed before output. The original data sequence to be transmitted is converted into a corresponding impulse chain firstly so as to control an electronic switch $s$. By (1), this chain always stays at low level (logic " 0 ") except for the beginning of bit " $M$ " $(M>0)$ and during the interval of $K T$ where the impulse appears and stays at high level (logic "1"). Controlled by this information impulse chain, the $s$ is connected to $s_{0}$ at low level, to $s_{1}$ at high level.

2.2. MPPSK Demodulation. The impacting filter (IF) is a special digital infinite impulse response (IIR) filter, with the feature of "notch-frequency selection" in an extremely narrow pass-band [6]. It highlights the difference of the modulation waveform, which is helpful for demodulation, and simplifies the structure of the receiver greatly. At present, the IF is artificially designed. In the following real simulation, we assume the impacting filter formed by one pair of conjugate zeros and four pairs of adjacent conjugate poles. The expression and related parameter of the IF is given in [10]. Consider

$$
H(z)=\frac{1+b_{1} \cdot z^{-1}+z^{-2}}{1-\sum_{i=1}^{2 n} a_{i} \cdot z^{-i}},
$$

where $n$ is the pair number of the conjugate poles. In order to demodulate dual-frequency MPPSK signals, the zero parameters of the IF are selected as $b_{1}=-1.6181733185991785$, and the pole parameters of the IF in this paper are selected as

$$
\begin{array}{ll}
a_{1}=-6.1150669443734404, & a_{2}=17.593270854070781, \\
a_{3}=-30.66190141963812, & a_{4}=35.258220132970798, \\
a_{5}=-27.343924194038685, & a_{6}=13.991777506187015, \\
a_{7}=-4.3370740838799371, & a_{8}=0.63250878596652416 .
\end{array}
$$

The proposed filter has a very narrow bandwidth, and the IF would retain the signal characteristics and reduce the noise. When MMPSK modulated signals pass the impacting filter, the special impacting filter can transform the tiny waveform difference into amplitude impacting, and Figure 3 would illustrate this demodulation process.

The coefficient for the $x$ axis is the index of a certain sample point. Set MPPSK Modulation parameter $M=4$. Waveform of a source symbol sequence $\{3,2,1,0\}$ is shown in Figure 3, Figure 3 illustrates its 4PPSK waveform after the modulation, Figure 3 depicts the response of this filter to 4MPSK modulated signals, the phase hopping can be converted into amplitude impacting, and its envelope is shown in Figure 3. 


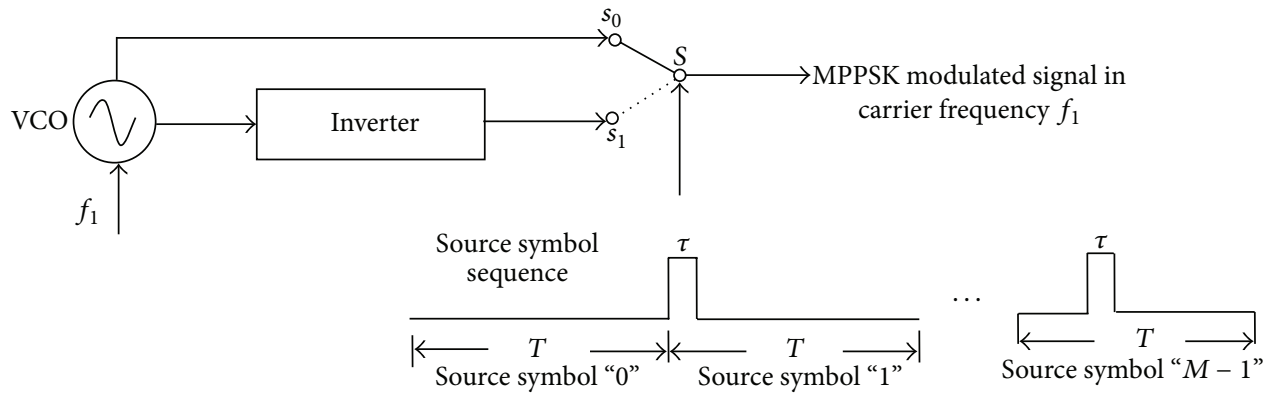

FIGURE 2: Block diagram of pulse train MPPSK modulator.
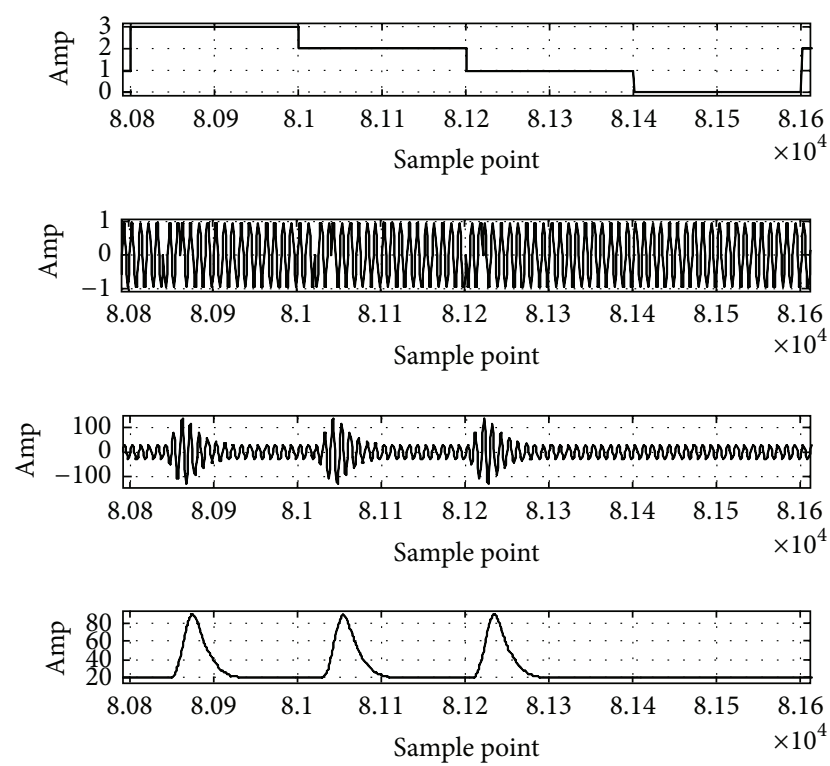

FIGURE 3: Block diagram of source, modulated signal, amplitude impacting, and envelope of amplitude impacting.

Nonzero symbols, that is, "1" to " $M-1$ ", can be distinguished from the different positions of the peak of amplitude impacting. Figure 3 shows the waveform corresponding to four symbols, “3”, " 2 ", "1", and "0." The modulation waveform for symbol "0" is a sinusoidal. Therefore, no impacting is observed in its response. The peak of amplitude impacting (at about $8.123 \times 10^{4}$ in Figure 3) corresponding to code "1" is close to the starting location (at about $8.12 \times 10^{4}$ in Figure 3). The followed modulation waveform is for code"2" (at about $8.106 \times 10^{4}$ in Figure 3), which is a little further from its own starting location (at about $8.10 \times 10^{4}$ in Figure 3). And the last is for code " 3 " (at about $8.088 \times 10^{4}$ in Figure 3), which is the furthest from its own starting location (at about $8.08 \times 10^{4}$ in Figure 3). Obviously, a simple amplitude threshold detector and bit synchronization can perform the demodulation for MPPSK signals, which results in the simple receiver structure. The impacting filter can be digitally implemented, which is beneficial for chip integration.

\section{Dual Frequency CW Ranging}

Dual frequency CW ranging technique can compute very adequate fine range resolution [11, 12], without using frequency modulation. Consider a CW signal of single frequency first:

$$
s_{t 0}(t)=\sin \left(2 \pi f_{0} t\right),
$$

where $f_{0}$ is the carrier frequency and $f_{0}=1 / T$. The receiver's range $R$ and is computed by measuring the time delay $t_{d}$, it takes CW signal to travel the path between transmitter and receiver. Since electromagnetic waves travel at the speed of light $c$, the received signal is

$$
s_{r 0}(t)=\sin \left[2 \pi f_{0}\left(t-t_{d}\right)\right]=\sin \left(2 \pi f_{0} t-\varphi\right),
$$

where the time delay $\varphi=2 \pi f_{0}(R / c)$.

Solving for $R$, we obtain

$$
R=\frac{c \varphi}{2 \pi f_{0}}=\frac{\lambda}{2 \pi} \varphi .
$$

Such a system with zero-intermediate frequency (ZIF) receiver is shown in Figure 4. PA stands for power amplifier and LNA represents low-noise amplifier; I/Q orthogonal channel is used in radar receiver for obtaining echo reflected signal phase and amplitude information. Clearly, the maximum unambiguous range occurs when $\varphi$ is maximum; that is, $\varphi=2 \pi$. Consider a system with two CW signals, denoted by $s_{t 0}(t)$ and $s_{t 1}(t)$, respectively. More precisely,

$$
s_{t 0}(t)=\sin \left(2 \pi f_{0} t+\phi_{0}\right), \quad s_{t 1}(t)=\sin \left(2 \pi f_{1} t+\phi_{1}\right) .
$$

The received signals from transmitter are

$$
\begin{aligned}
& s_{r 0}(t)=\sin \left(2 \pi f_{0} t+\phi_{0}+\varphi_{0}\right), \\
& s_{r 1}(t)=\sin \left(2 \pi f_{1} t+\phi_{1}+\varphi_{1}\right),
\end{aligned}
$$

where $\varphi_{0}=2 \pi f_{0} R / c$ and $\varphi_{1}=2 \pi f_{1} R / c$.

After heterodyning (mixing) with the carrier frequency, the phase difference between the two received signals is

$$
\Delta \varphi=\frac{2 \pi f_{0} R}{c} \Delta f_{10},
$$

where $\Delta \varphi=\varphi_{1}-\varphi_{0}$ and $\Delta f_{10}=f_{1}-f_{0}$. 


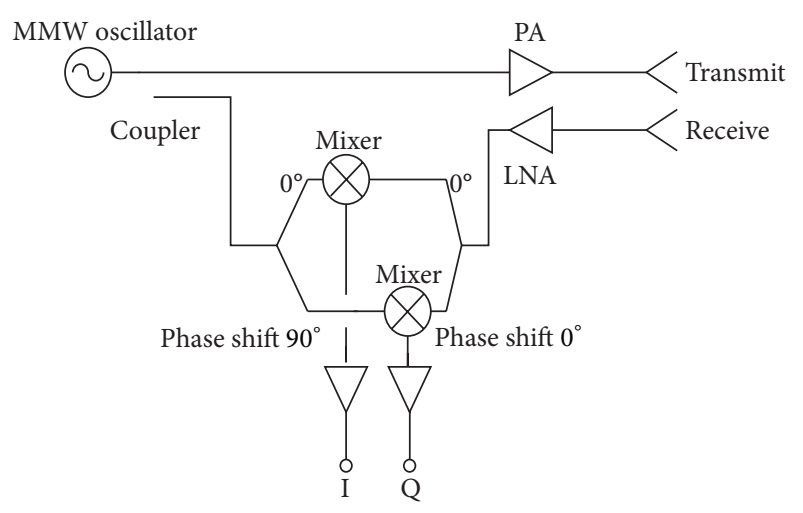

FIGURE 4: Block diagram of a CW Ranging system with single frequency.

Again $R$ is maximum when $\Delta \varphi=2 \pi$; however, at this circumstance, the maximum unambiguous range is

$$
R_{10 \max }=\frac{c}{\Delta f_{10}} .
$$

In practice, besides the carrier frequency difference $\Delta f_{10}$, range resolution depends on SNR [13], approximately $1 / 10$ wavelength, which is larger than the ideally achievable value. Then the range resolution $\delta R_{10}$ can be

$$
\delta R_{10}=\frac{c}{2 \pi \Delta f_{10} \sqrt{2 \cdot \mathrm{SNR}}} .
$$

Ambiguity may occur in range measurement especially when carrier frequency difference $\Delta f_{10}$ is high or the receiver's range is longer than $R_{10 \text { max }}$. From (10) and (11), achieving fine range resolution can be accomplished by increasing frequency different $\Delta f_{10}$, but maximum unambiguous ranging $R_{10 \text { max }}$ is decreasing. The limited ranging measurement also limits the actual application value of dual frequency $\mathrm{CW}$ radar.

\section{Dual Frequency MPPSK System Model}

4.1. Broadcasting and Ranging Transmitter. Broadcasting and ranging transmitter equipment is mainly composed of two pulse train MPPSK modulation generators, as shown in Figure 5. PA stands for power amplifier.

Two working modes are designed which depend on the original data source. For digital video broadcasting, the data rate and the bit error rate (BER) are the most stringent parameters. Pulse train MPPSK modulator 1 transmits MPPSK signals, which is efficient regarding the amount of information they convey on the bandwidth they occupied. And pulse train MPPSK modulator 2 will stop working when satellite is particularly affected by power limitations.

For the ranging function, the waveform should be robust against interference, noise, and distortion due to multipathpropagation. The pulse train MPPSK modulator 1 transmits MPPSK signals, which are modulated by the original data symbol " 1 " constantly and is sine-like waveform. Pulse train

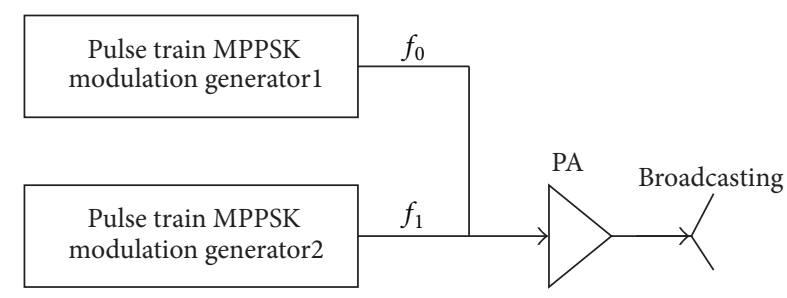

FIGURE 5: Block diagram of broadcasting transmitter equipment.

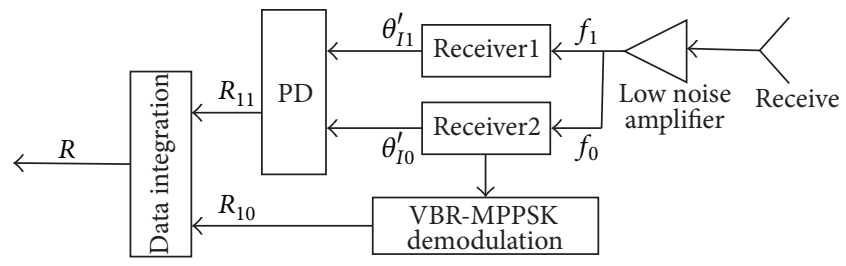

FIGURE 6: Block diagram of dual frequency demodulation receiver.

MPPSK modulator 2 transmits CW, which may be considered to be a special kind of MPPSK signals, the system signals are denoted by $s_{t 01}(t)$.

Consider

$s_{t 0}(t)=\left\{\begin{array}{lc}\sin \left(2 \pi f_{0} t\right) & 0 \leq t<N T, k=0, \\ \sin \left(2 \pi f_{0} t\right) & 0 \leq t \leq(k-1) K T, \\ -\sin \left(2 \pi f_{0} t\right) & (k-1) K T<t<k K T, \\ \sin \left(2 \pi f_{0} t\right) & 0 \leq t \leq \pi, 1 \leq k \leq M-1, \\ k K T \leq t<N T,\end{array}\right.$

$s_{t 1}(t)=\sin \left(2 \pi f_{1} t\right)$,

$s_{t 01}(t)=s_{t 0}(t)+s_{t 1}(t)$,

where $f_{0}$ and $f_{1}$ are the carrier frequency, and for $s_{t 0}(t)$, symbol rate is $R$, which is denoted by

$$
R=\frac{f_{0} \log _{2} M}{N} .
$$

The power spectral density (PSD) of $s_{t 01}(t)$ has been figured out in conditions of " $M-1$ " distributed in equal probability.

Consider

$$
\begin{aligned}
P_{r 1}(f)=f_{s}\left[\frac{1-M}{M^{2}}\left|G_{M-1}(f)-G_{0}(f)\right|^{2}\right. & \\
& +\cdots+\frac{1-M}{M^{2}}\left|G_{M-1}(f)-G_{M-2}(f)\right|^{2} \\
& -\sum_{i=0}^{M-2} \sum_{j=1+i}^{M-1} \frac{1}{M^{2}}\left|G_{M-1}(f)-G_{i}(f)\right| \\
& \left.\times\left|G_{M-1}(f)-G_{j}(f)\right|\right]
\end{aligned}
$$




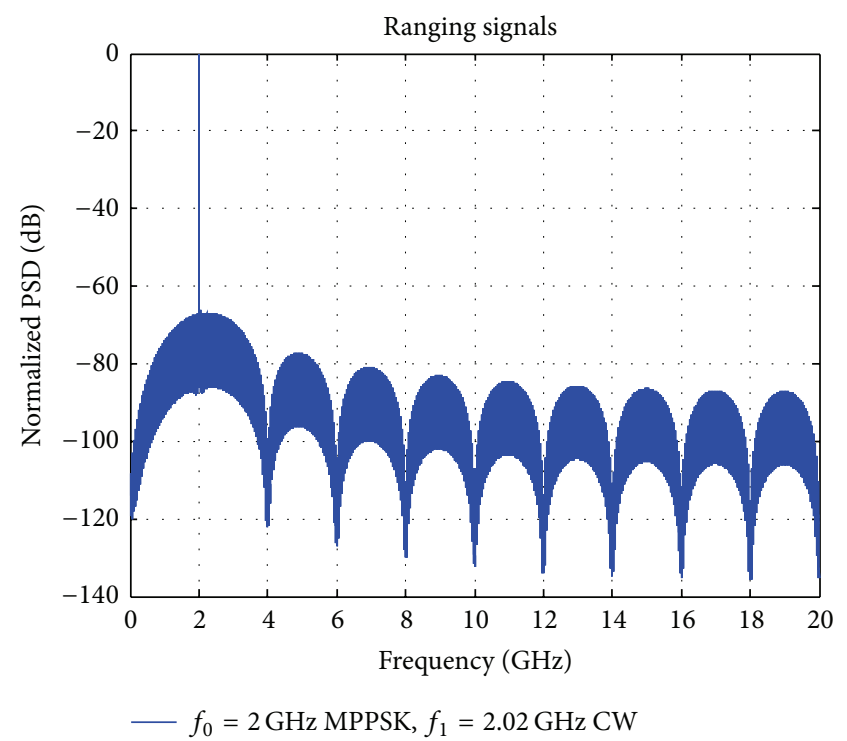

(a)

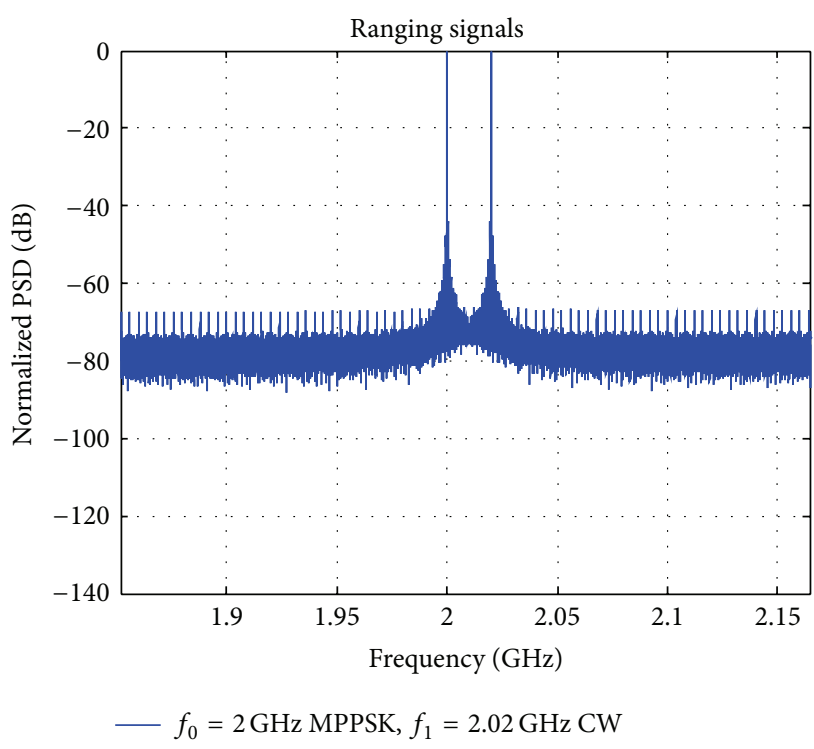

(b)

FIGURE 7: The PSD of MPPSK ranging signal.

$$
\begin{aligned}
& +\left(\frac{f_{s}}{M}\right)^{2} \sum_{m=-\infty}^{\infty} \delta\left(f-m f_{s}\right) \\
& +\mid G_{0}\left(m f_{s}\right)+G_{1}\left(m f_{s}\right) \\
& +\cdots+\left.G_{M-1}\left(m f_{s}\right)\right|^{2} \\
& +\cdots \\
& +\frac{1}{4} \delta\left(f \pm f_{1}\right),
\end{aligned}
$$

where $f_{s}=f_{0} / N, G_{0}(f), G_{1}(f) \cdots G_{M-1}(f)$ is Fourier transform of MPPSK modulated waveforms corresponding to symbol “0”, “1”, .., “ $M-1$ ”, respectively.

4.2. Broadcasting and Ranging Receiver. Broadcast and ranging reception equipment is made by two kinds of receivers, and one is mainly composed of phase discriminator (PD), and the other is mainly composed of variable bit rate MPPSK (VBR-MPPSK) demodulation. As shown in Figure 6, receiver 1 and receiver 2 have been illustrated in Figure 4, and principle of MPPSK demodulation has been described in Section 2. The MPPSK signal in carrier frequency $f_{0}$ is demodulated by VBR-MPPSK demodulation. The output data $R_{10}$ can be converted to video after data processing. Signals both in carrier frequency $f_{0}$ and in carrier frequency $f_{1}$ are demodulated with PD. The output data $R_{11}$ combined with $R_{10}$ would be converted to distance value after data fusion.

Not only the employed waveform but also the general parameters have to be chosen according to the requirements derived from both applications. For broadcasting, digital video signals are demodulated by VBR-MPPSK demodulator alone. From (1), the variables $M, K$ and $N$ form a parameter set to adjust the bandwidth, data rate and BER performance. Increasing value of $M$ can lead to higher data because more time slots are utilized. While taking multipath channel environment into consideration, large $N$ is advantageous in mitigating multipath effect but pulls down the data rate and the efficiency of time slot. Therefore, small values of $K / N$ results in sine-like waveform, which presents in solitudepeak low-sideband PSD apearence and occupies very limited bandwidth. So it is very important to trade off the parameters selection of MPPSK.

For ranging, theoretically, the maximum unambiguous range of MPPSK system must correspond to $N$, which is denoted by

$$
R_{\max }=c T_{0}=c \frac{N}{f_{0}},
$$

and $K$ should be chosen according to $\Delta f_{10}$ as

$$
R_{10 \max }=\frac{c}{\Delta f_{10}}=c \frac{K}{f_{0}} .
$$

So the maximum unambiguous ranging $R_{11 \max }$ of dual frequency CW ranging system would be increased $m$ times as follows:

$$
R_{\max }=m R_{11 \text { max }} .
$$

Combining (15) and (16) yields

$$
N \geq m \frac{f_{0}}{\Delta f_{10}},
$$

From Figure 6, $R_{11}$ is high-precision measured value, and $R_{10}$ is wide-range measured value. Combining the two measured values, the system would output a value of $R$ with highprecision and wide-range; that is,

$$
R=\left\lceil\frac{R_{10}}{R_{11 \text { max }}}\right\rceil R_{11 \text { max }}+R_{11},
$$

where \lceil\rceil stands for round up. 


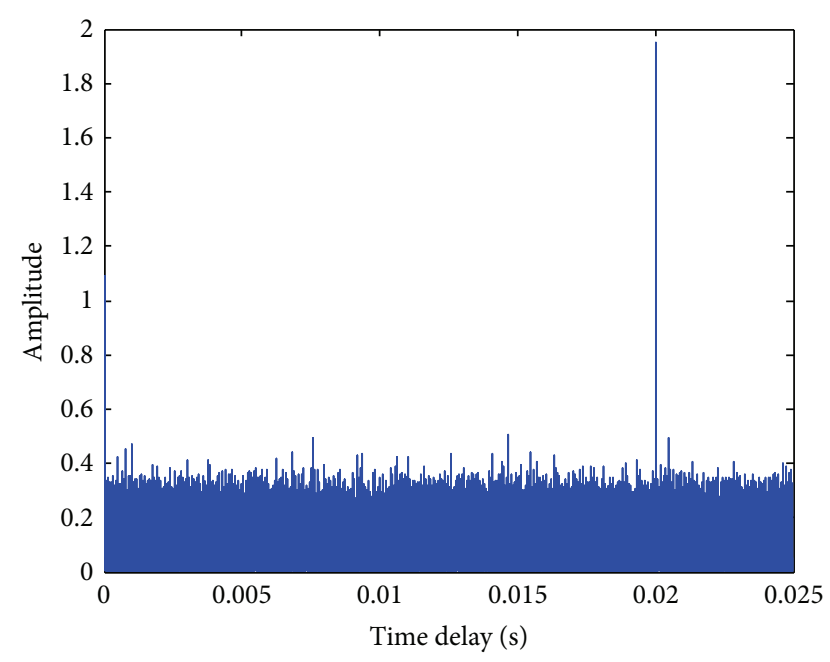

FIGURE 8: Simulation the rough range value.

TABLE 1: Simulation parameter for ranging.

Parameter value

Carrier frequency: $f_{0}=2 \mathrm{GHz} f_{1}=2.02 \mathrm{GHz}$

Modulation: $f_{0}$ : MPPSK, $f_{1}$ : CW

Modulation parameter: $K=100, N=5 \times 10^{6}, M=2$

Receiver distance: $600 \mathrm{~km}$

Due to the increase in carrier frequency $f_{1}$ of a CW signal, the dual frequency MPPSK signal becomes synthetical waveforms, that are suitable, respectively, for performing both data broadcasting and ranging. Compared with dual frequency $\mathrm{CW}$ ranging technique, the maximum unambiguous range is extended from $c / \Delta f_{10}$ to $N\left(c / f_{0}\right)$.

\section{Simulation Results}

5.1. Ranging Mode. In this section, the performance of the proposed dual frequency MPPSK system is simulated. Firstly, we consider the dual frequency MPPSK system shown in Figures 5 and 6 in ranging mode. In Table 1, a summary of the most important parameters of the simulation model is provided.

As shown in Figure 7, the left subfigure is the global graph and the right one is the local enlarging graph for the carrier frequencies $f_{0}$ and $f_{1}$. Obviously, the PSD of MPPSK ranging signal has a more narrow bandwidth with $99 \%$ power (or $-60 \mathrm{~dB}$ bandwidth). When $K / N$ turns small, dual frequency MPPSK signal becomes more similar to pure sine waveform. Spectra of the ranging signal from system will be concentrated at carrier frequency $f_{0}=2 \mathrm{GHz}$ and carrier frequency $f_{1}=2.02 \mathrm{GHz}$. Smaller portion of the sine carrier can be changed, and the line spectrum components in PSD of the MPPSK modulated signal become lower and even disappear. MPPSK tries to allocate as much power as possible to the carrier.

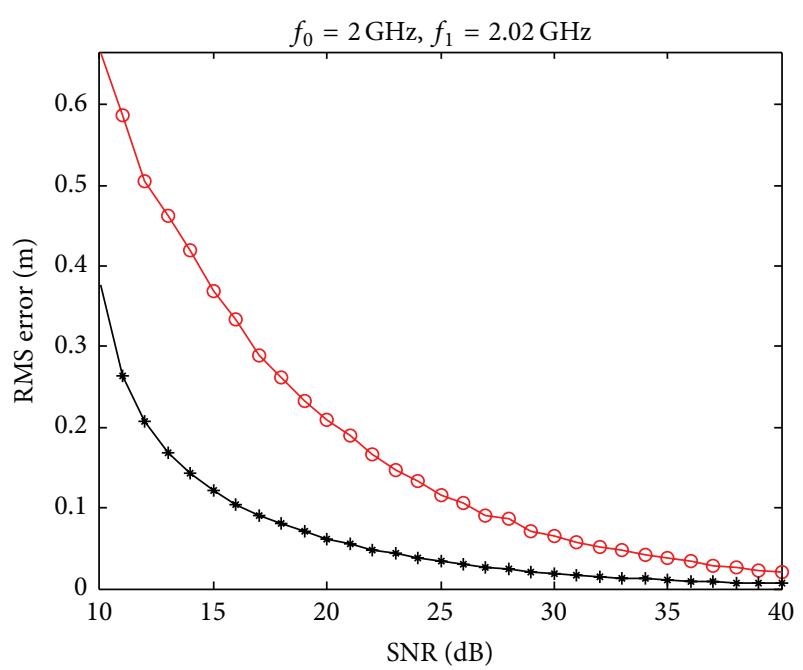

- Simulation results

$\rightarrow$ C-R bound

FIGURE 9: The simulation result and C-R bound.

The simulation experiment is in the AWGN channels $(\mathrm{SNR}=2 \mathrm{~dB})$, and as shown in Figure 8 , the result is output of VBR-MPPSK demodulation. The rough range value can be computed by the time delay corresponding to amplitude impacting. The amplitude impacting is at the time delay of $1.9997 \mathrm{~ms}$, and from (15), the rough range value $R_{10}$ is 599 $910 \mathrm{~m}$.

From Figure 9, the simulation result and C-R bound just have approximately $0.3 \mathrm{~m}$ difference when SNR is $10 \mathrm{~dB}$, with the increase of SNR, difference between simulation result and the theoretical result decreases, and RMS error shrinks towards equality in $\mathrm{SNR}=40 \mathrm{~dB}$.

5.2. Broadcasting Mode. We also consider the system shown in Figures 5 and 6 in broadcasting mode. In Table 2, a summary of the most important parameters of the simulation model is provided.

Figure 10 shows the PSD of QPSK and proposed MPPSK signal with the same modulation parameters. As shown in Figure 10, the left subfigure is the global graph of the PSD of 8 PPSK and QPSK, and right one is the local enlarged graph nearing the carrier frequency $f_{0}$. Obviously, in MPPSK modulation, the line spectra, illustrating the periodic components of the modulated signal, decrease greatly, because of the random choosing of the $M-1$ positions. Therefore, MPPSK becomes more approximate to sine signal, the bandwidth with $99 \%$ power (or with $-60 \mathrm{~dB}$ bandwidth) decreases, and the spectra efficiency is improved greatly.

Figure 11 illustrates the SER in different modem, and the simulation shows that at SER $=10^{-4}$, the SNR performance of the system with MPPSK modem may be improved by approximately $8 \mathrm{~dB}$ and $13 \mathrm{~dB}$ as compared with the performance of DVB-S with QPSK modulation and coherent demodulation and 16-QAM modulation and coherent demodulation, respectively. 


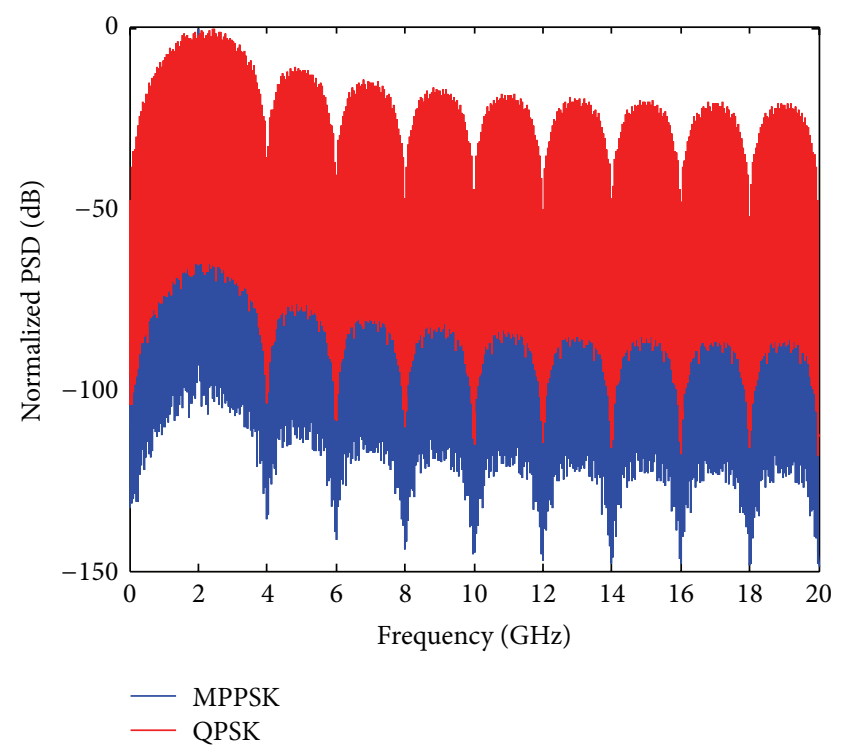

(a)

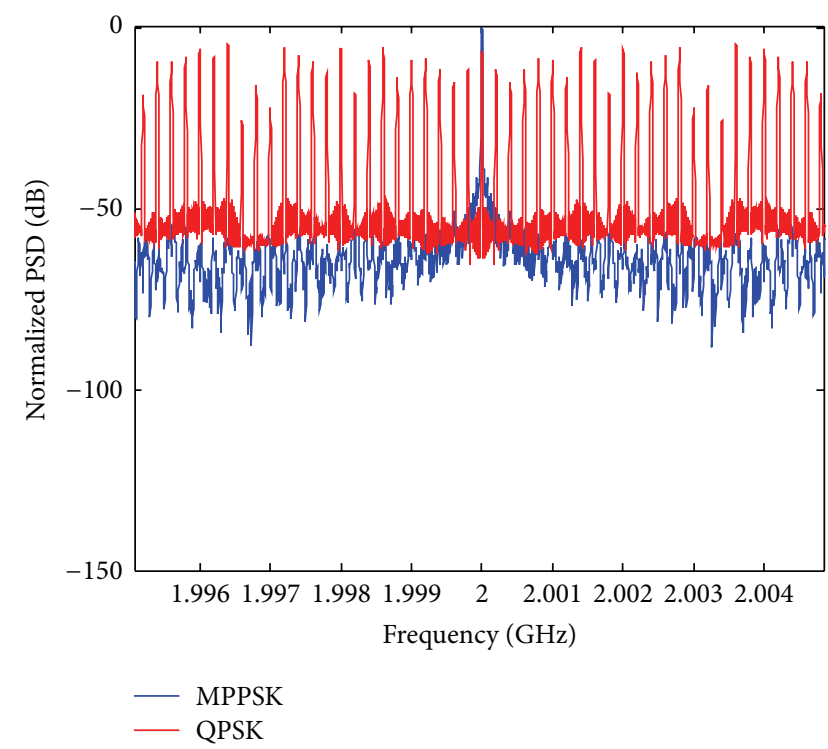

(b)

FIGURE 10: The PSD of 8PPSK and QPSK.

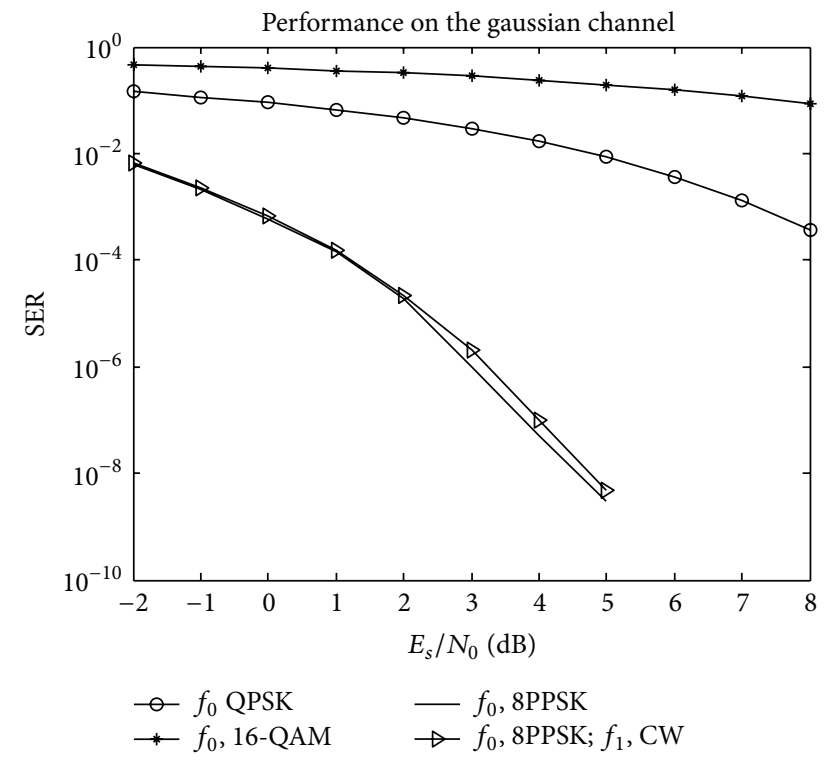

FIGURE 11: Comparative SER results of 8 PPSK, dual frequency 8 PPSK, QPSK and 16-QAM.

The result of the dual frequency MPPSK system and single frequency MPPSK system just have less than $1 \mathrm{~dB}$ difference in order to obtain the same SER performance, which is caused by the detection method and the selection of the decision threshold in the simulation. Simultaneously, research on the optimal modem method is underway; the system performance still has room for improvement.

\section{Conclusions}

The SER performance of the dual frequency MPPSK system is better than that of QPSK in such case, and its occupied
TABLE 2: Simulation parameters for broadcasting.

Parameter value

Carrier frequency $f_{0}=2 \mathrm{GHz}$

Modulation $f_{0}$ : MPPSK

Modulation parameters: $K=2, N=20, M=8$

bit rate: $300 \mathrm{Mbps}$

bandwidth is much narrower than QPSK. QPSK tries to spread as much power as possible to the sidebands. On the opposite side, MPPSK allocates most power in the carrier to keep sideband energy emissions negligible, and dual frequency MPPSK system also provides an augmentation service, that is, the ranging function. After data integration between PD receiver and VBR-MPPSK demodulator, system would provide high precision ranging and extend maximum unambiguous range as well. The dual frequency MPPSK system is illustrated in block diagram, which is advantageous MPPSK signal generator and integrated receiver architecture, PD and impacting filter that essentially determine dual frequency signal demodulation are emphasized in the paper and correlation receiver distance simulation are made. The future work will continue with the research on dual frequency MPPSK system, including selection and combinations of other impacting filter and ranging algorithms. Different channel environment profile, as well as channel coding will be further considered.

\section{Acknowledgments}

The authors thank all of the reviewers for their valuable comments, which have considerably helped in improving the overall quality of the work presented in the revised paper. 
This work was supported by the National Natural Science Foundation of China (61271204).

\section{References}

[1] ETSI, "Digital broadcasting systems for television, sound and data service, framing structure, channel coding and modulation for 11/12 GHz satellite services," ETS 300 421, 1994.

[2] C. Qi and L. Wu, "PLL demodulation technique for M-ray position phase shift keying," Journal of Electronics, vol. 26, no. 3, pp. 289-295, 2009.

[3] P. K. Ying and L. N. Wu, "New scheme of MPPSK modem," Journal of Southeast University, vol. 42, no. 2, pp. 204-208, 2012.

[4] L. Wu and M. Feng, "On BER performance of EBPSK-MODEM in AWGN channel," Sensors, vol. 10, no. 4, pp. 3824-3834, 2010.

[5] J. Sun, W. Fang, and W. Xu, "A quantum-behaved particle swarm optimization with diversity-guided mutation for the design of two-dimensional IIR digital filters," IEEE Transactions on Circuits and Systems II, vol. 57, no. 2, pp. 141-145, 2010.

[6] M. Feng, L. Wu, and P. Gao, "From special analogous crystal filters to digital impacting filters," Digital Signal Processing, vol. 22, no. 4, pp. 690-696, 2012.

[7] P. van Genderen, "Recent advances in waveforms for radar, including those with communication capability," in Proceeding of the European Radar Conference (EuRAD '09), pp. 318-325, Rome, Italy, October 2009.

[8] C. Sturm and W. Wiesbeck, "Waveform design and signal processing aspects for fusion of wireless communications and radar sensing," Proceedings of the IEEE, vol. 99, no. 7, pp. 12361259, 2011.

[9] Y. Zhang, M. Amin, and F. Ahmad, "A novel approach for multiple moving target localization using dual-frequency radars and time-frequency distributions," in Proceeding of the 41st Asilomar Conference on Signals, Systems and Computers (ACSSC '07), pp. 1817-1821, Pacific Grove, Calif, USA, November 2007.

[10] M. Feng, L. Wu, J. Ding, and C. Qi, "BER analysis and verification of EBPSK system in AWGN channel," IEICE Transactions on Communications, vol. E94-B, no. 3, pp. 806-809, 2011.

[11] Weibel Inc, "Weibel RR-60034 ranging radar system".

[12] Weibel, "Weibel azimuth \& elevation monopulse doppler tracking radar systems," 1997-2002.

[13] S. Peleg and B. Porat, "The Cramer-Rao lower bound for signals with constant amplitude and polynomial phase," IEEE Transactions on Signal Processing, vol. 39, no. 3, pp. 749-752, 1991. 


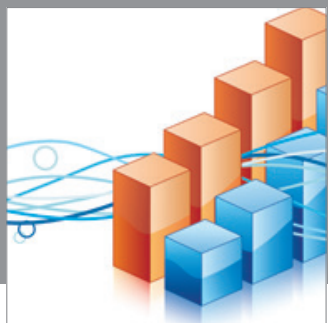

Advances in

Operations Research

mansans

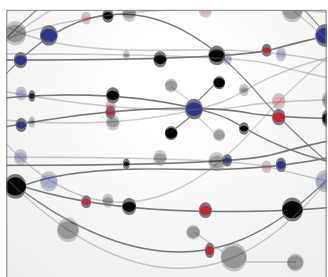

The Scientific World Journal
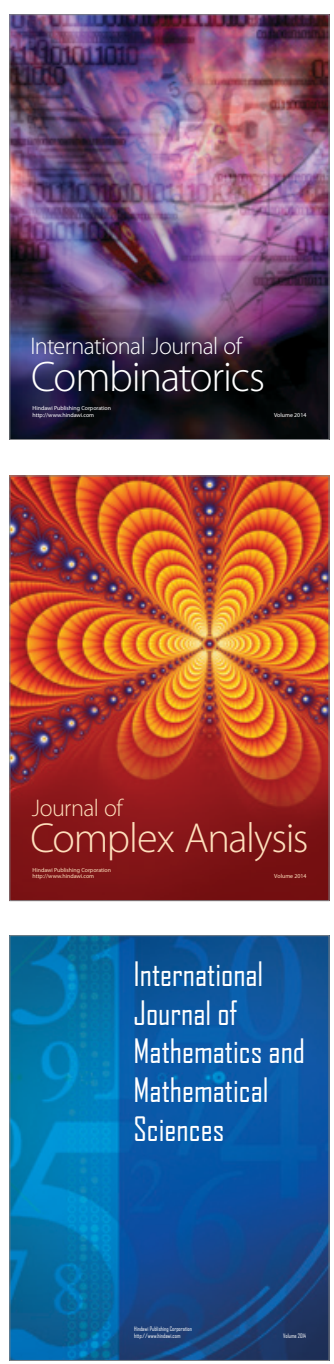
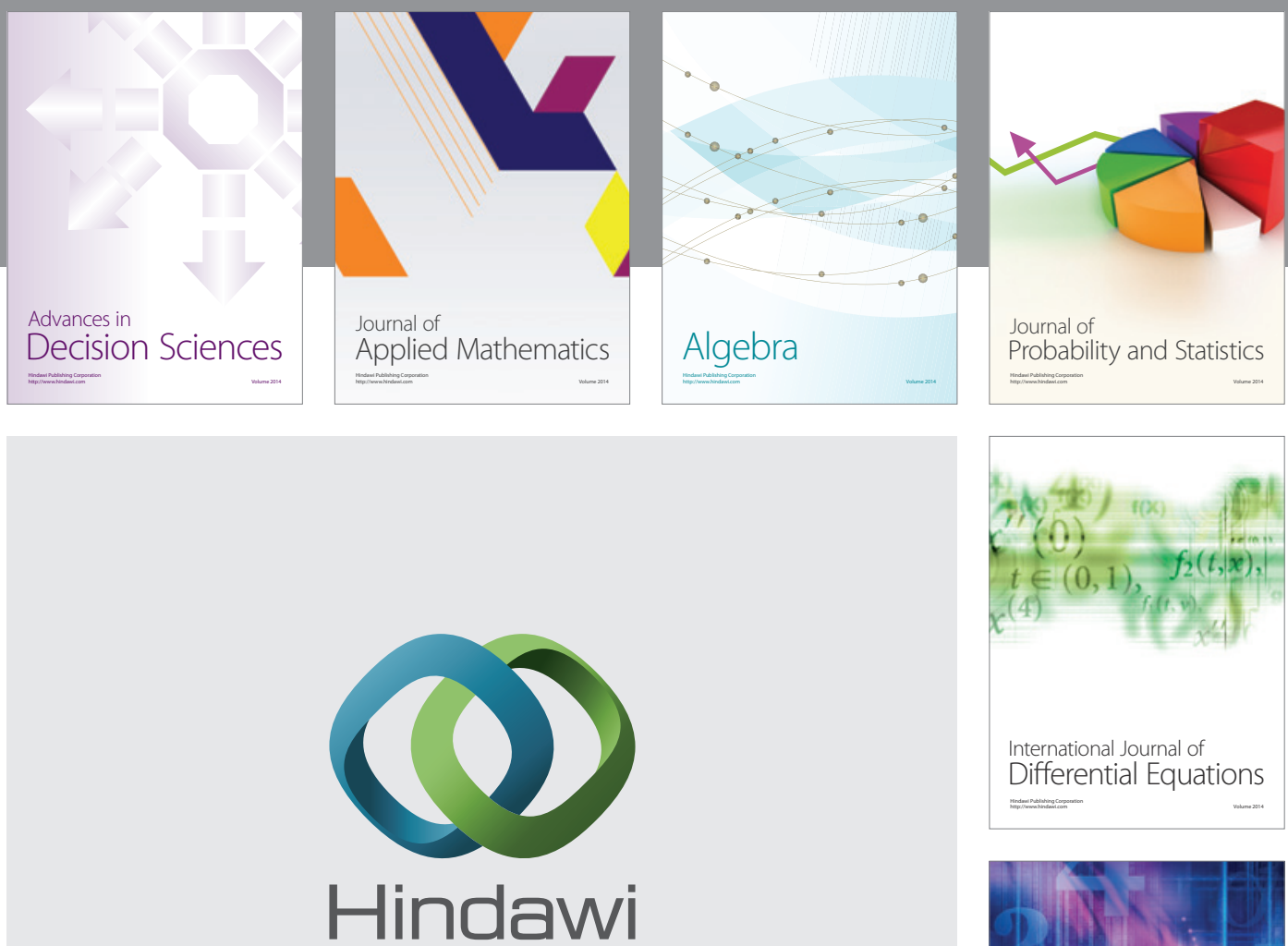

Submit your manuscripts at http://www.hindawi.com
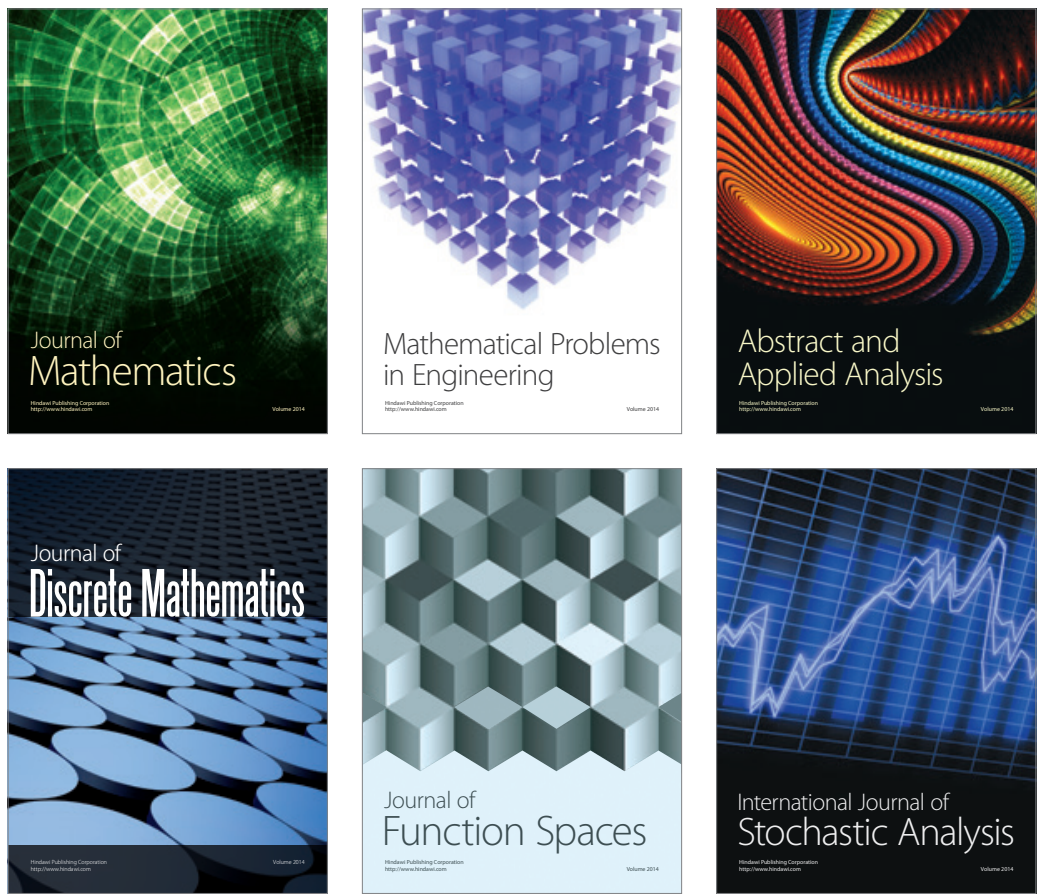

Journal of

Function Spaces

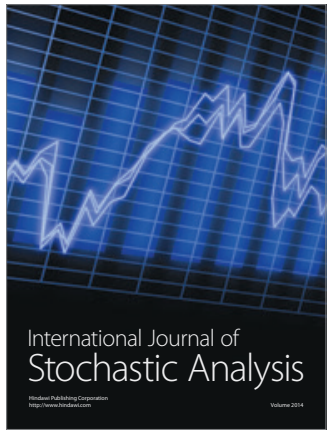

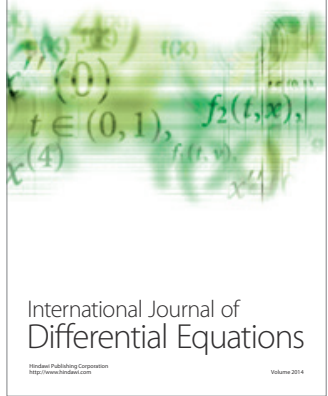
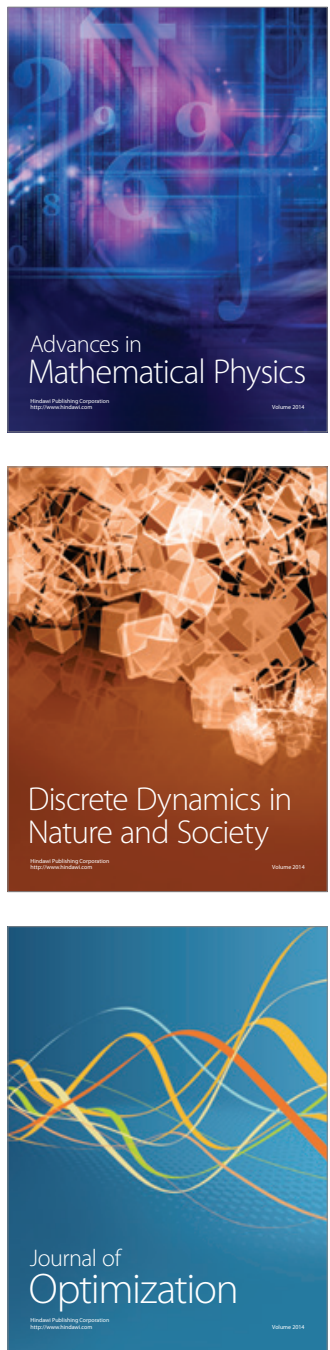